\title{
PRAGMATISMO, DESIGN-BASED RESEARCH E INVESTIGAÇÃO-AÇÃO
}

\section{PRAGMATISM, DESIGN-BASED RESEARCH AND ACTION RESEARCH}

\author{
Alda Pereira ${ }^{1}$ \\ Isolina Oliveira ${ }^{2}$
}

\begin{abstract}
Resumo: Neste artigo as autoras analisam e refletem sobre as caraterísticas de duas abordagens ligadas a uma epistemologia da prática: Design-Based Research e Investigação-Ação e o seu enquadramento no Paradigma Pragmático da investigação em Educação. A Design-Based-Research engloba um conjunto de abordagens com o objetivo de produzir novas teorias, artefactos e práticas com impacto na aprendizagem em contextos naturais; a Investigação-Ação é orientada para a ação ou ciclos de ações que os membros de uma organização/comunidade, podendo incluir constituintes externos, desenvolvem em torno de uma situação problemática visando o melhoramento de práticas. As raízes e a evolução das duas abordagens são distintas, porém partilham assunções fundamentais e traduzem olhares investigativos sobre a prática com subtis diferenças. A análise dos respetivos traços ontológicos, epistemológicos e metodológicos a elas subjacentes justificam a sua inclusão no Paradigma Pragmático de Investigação em Educação.
\end{abstract}

Palavras-chave: Design-Based Research; Investigação-Ação; Pragmatismo.

\begin{abstract}
In this article the authors analyse and reflect on the characteristics of two approaches linked to an epistemology of practice: Design-Based Research and Action-Research and their framework in the Pragmatic Paradigm of research in Education. Design-Based Research encompasses a set of approaches aimed at producing new theories, artefacts, and practices with an impact on learning in natural contexts; Action-Research is action-oriented or cycles of actions that members of an organisation/community, possibly including external constituents, develop around a problematic situation aimed at improving practices. The roots and evolution of the two approaches are distinct, but they share fundamental assumptions and translate investigative views on practice with subtle differences. The analysis of the respective ontological, epistemological and methodological features underlying them justifies their inclusion in the Pragmatic Paradigm of Research in Education.
\end{abstract}

Keywords: Design-Based Research; Action Research; Pragmatism.

\section{Introdução}

Quando Denzin \& Lincoln (1994) discutem os paradigmas de investigação teoria crítica pós-moderna e construtivismo - fazem-no em busca de legitimidade face à hegemonia do paradigma positivista ou pós-positivista que então dominava a investigação. Havia um interesse crescente pelas questões ontológicas e epistemológicas nos estudos realizados no campo das ciências sociais. Os autores propõem, então, 4

\footnotetext{
${ }^{1}$ Doutora em Ciências da Educação pela Universidade Aberta, Portugal. Investigadora sénior integrada no Laboratório de Educação a Distância e eLearning, Universidade Aberta, Lisboa, Portugal. E-mail: Alda.Pereira@uab.pt

${ }^{2}$ Doutora em Ciências da Educação pela Universidade de Lisboa (ULisboa) - Portugal. Investigadora sénior no Laboratório de Educação a Distância e Elearning, Universidade Aberta (LE@D- UAb). Lisboa, Portugal. E-mail: Isolina.Oliveira@uab.pt
} 
paradigmas - positivismo, pós-positivismo, teoria crítica e construtivismo - distintos pelo tipo de ontologia, epistemologia e metodologia em que cada um se situa e com base num conjunto de critérios.

Mais tarde, Guba e Lincoln (2005) discutem as controvérsias em torno dos quatro paradigmas referidos, acrescentando o paradigma participatório caraterizado do ponto de vista epistemológico pela subjetividade crítica e metodologicamente pela participação política na investigação de ação colaborativa, com a primazia do saber prático. Importa ter presente que, tal como afirmam os autores, os limites dos paradigmas são fluídos e passíveis de mudança.

Desde então, a discussão em torno dos paradigmas de investigação tem vindo a evoluir numa tentativa de maior precisão na definição de critérios, incorporando outros conceitos e, também, questionando a caraterização dos próprios paradigmas. Destaca-se sobretudo a controvérsia que marcou a dualidade quantitativo/qualitativo, caraterizadora dos paradigmas positivista/interpretativo e a sua dificuldade na aplicação a investigação realizada fora destes enquadramentos. Importa considerar as questões advindas da inovação tecnológica e das novas possibilidades em educação com o uso das tecnologias digitais, como se observa na atual pandemia, as quais suscitam questionamentos e designs de investigação que nem sempre são enquadráveis nos atuais paradigmas.

A pluralidade de situações, contextos, cenários, compreensíveis pelas interações geradas, e de métodos obrigam-nos a questionar e a repensar os estudos realizados na área da Educação no que diz respeito aos paradigmas que os configuram (PEREIRA; OLIVEIRA, 2020). Os estudos por nós realizados e/ou orientados estão ligados à prática profissional, situam-se em contextos interativos específicos, envolvem vários métodos e têm como objetivo a introdução de mudança e inovação nos locais de trabalho, enquadrando-se no design de investigação-ação ou design based research.

Neste panorama, o presente artigo organiza-se tomando como ponto de partida a sistematização dos vários paradigmas de investigação incluindo as asserções do conhecimento pragmático, seguido da reanálise do design-based research e da investigação-ação, considerando as suas raízes epistemológicos, traços distintivos e desafios emergentes para identificar e compreender possíveis convergências. Numa síntese final, argumenta-se sobre a inclusão do Design-Based Research e da InvestigaçãoAção no Paradigma Pragmático. 


\section{Paradigmas de investigação em contextos educacionais}

Os paradigmas são instrumentos conceptuais e práticos que usamos para enquadrar e resolver problemas no âmbito da investigação (KAUSHIK; WALSH, 2019). O conceito de paradigma tem tido diferentes interpretações desde que Thomas Kuhn o apresentou na publicação The Structure of Scientific Revolutions associado a um modo de pensamento filosófico mas também ligado a exemplo, no sentido de modelo de investigação (GASPAR et al., 2015).

O termo paradigma é usado para partilhar crenças, assunções, valores e práticas num dada comunidade científica (CRESWELL, 1998; MORGAN, 2007) e é visto como um exemplo modelo sobre como a investigação deve ser feita numa determinada área. $\mathrm{Na}$ investigação em contextos educacionais um paradigma refere a visão do investigador sobre o mundo, ou seja, as suas crenças e princípios que orientam o modo como ele perspetiva, interpreta e atua, nomeadamente como informa o significado e a interpretação dos dados. Traduz, pois, um pensamento, uma escola de pensamento, que orienta/subjaz a um dado campo de investigação. Os paradigmas são perspetivas, escolas de pensamento historicamente contextualizados, contudo, pela sua capacidade explicativa, um dado paradigma pode ser reconhecido noutros contextos desde que se adeqúe ao objeto de estudo (GASPAR et al., 2015). O termo paradigma tem vindo a ser usado, em linguagem corrente, como "quadro de referência" a partir do qual se discute, investiga e trabalha com problemas de diferente natureza.

Denzin e Lincoln (2004) realçam que os paradigmas são construções humanas e definem a visão do mundo do investigador. Apresentam quatro elementos na caraterização dos paradigmas: i) axiologia que implica a aceitação de um conjunto de valores, remetendo para as questões éticas a considerar no planeamento da investigação; ii) ontologia que suscita questões sobre a natureza da existência, a realidade existe fora do mundo social ou é uma construção criada pela mente, ou seja, a realidade é de natureza objetiva ou fruto da mente?; iii) epistemologia que remete para como se conhece o mundo, ou de outro modo, qual é a natureza do conhecimento?; iv) metodologia prende-se com o como se obtém conhecimento sobre o mundo, refere-se ao design do estudo, aos métodos e procedimentos usados na investigação, a qual é planeada para se responder a algo, isto é, como devo proceder para obter dados e conhecimentos que permitam responder às questões de investigação e contribuir para o conhecimento. 
O conceito de paradigma suscita várias controvérsias, ainda que seja consensual a definição de vários paradigmas com base nesses elementos caraterizadores. A sistematização de Denzin e Lincoln (1994) constitui uma referência e, neste sentido, com base nela agrupam-se em quatro os paradigmas de investigação em contextos educacionais: positivismo, interpretativo, crítico e pragmático.

O paradigma positivista tem origem no conceito de positivismo, atribuído a August Comte (1832-42), o qual é suportado no método científico, envolvendo a experimentação para explorar as observações, interpretá-las em termos de factos e obter resultados mensuráveis. Postula o conhecimento objetivo, generalizável independentemente do tempo e do contexto, e replicável de uma realidade externa que é independente do sujeito. Aceita que o sujeito do conhecimento é independente do objeto do conhecimento e a investigação é independente de valores. Pretende explicar e fazer previsões com base em resultados mensuráveis. São aspetos centrais neste paradigma o determinismo, pela relação causa-efeito e o empirismo pela relevância atribuída à recolha de dados empíricos que suportam a teoria escolhida e que permitem testar, aceitando ou rejeitando as hipóteses formuladas. Neste sentido, privilegiam-se os métodos quantitativos e metodologias de natureza experimental, quasi-experimental, correlacional, comparativa causal, por inquérito, pois possibilitam ao investigador ser preciso na apresentação e descrição dos dados recolhidos e explicar as relações que emergem da sua análise.

O paradigma interpretativo assume que a situação a estudar tem múltiplas realidades que podem ser construídas e reconstruídas. A proximidade entre o investigador e os participantes na investigação é centrada na construção de sentido. Os investigadores são parte da situação, ou seja, o investigador é um instrumento chave situado no contexto que está a estudar (BODGAN; BIKLEN, 1994), donde a importância atribuída à reflexividade do mesmo (WELLINGTON, 2000). Reconhece-se a subjetividade na análise dos dados que é interpretativa, indutiva e dependente do investigador. $\mathrm{O}$ conhecimento é construído socialmente a partir da experiência do investigador, na interação com os participantes e enraizado no contexto em que se situa. Assim, a teoria não precede a investigação mas antes tende a emergir dos dados gerados pela ato de investigar e os resultados refletem os valores do investigador, os quais devem ser explicitados. Não há o objetivo em compreender uma lei universal mas o indivíduo e, neste sentido, são privilegiados os métodos qualitativos e, entre outras, metodologias de estudo de caso, por narrativas, etnográficas, fenomenológicas e de investigação-ação. 
O paradigma crítico, também chamado de participatório ou transformativo, é usado sobretudo em situações de justiça social e pretende compreender as questões políticas, sociais e económicas e sustentar a intervenção com o objetivo de transformar uma dada situação. Por espoletar o debate político em torno destas questões e por promover a mudança é emancipatório pois contribui para a tomada de consciência de constrangimentos que limitam o desenvolvimento (KEMMIS; WILKINSON, 1998). Do ponto de vista ontológico assume o realismo histórico em que a realidade é moldada por valores políticos, culturais, económicos, étnicos e de género. Carateriza-se pela epistemologia transacional por o investigador interagir com os participantes e metodologicamente pela participação política na investigação de ação colaborativa, com alta confiança no saber prático (DENZIN; LINCOLN, 2000). Reconhecem-se as consequências em privilegiar certas versões da realidade, respeitam-se as normas culturais pelo que os resultados são mediados por valores e privilegiam-se, entre outras, metodologias associadas à investigação-ação, aos estudos culturais e feministas (CRESWELL, 2003).

O paradigma pragmático busca as suas raízes e fundamentação na filosofia do pragmatismo. Há diferentes entendimentos de pragmatismo, mas historicamente situa-se nos trabalhos de Peirce, James, Mead, and Dewey (CRESWELL, 2003; MORGAN, 2014). Do ponto de vista ontológico, o pragmatismo considera inútil a discussão em torno da "verdade" e da "realidade (CRESWELL, 2007). Aceita-se que não há uma realidade única, todos os indivíduos têm a sua, fazendo interpretações singulares, mas essa realidade está enraizada no ambiente e só pode ser conhecida através da ação (KAUSHIK; WALSH, 2019). A verdade é o que num dado momento funciona e não se baseia no dualismo entre uma realidade independente da mente ou dentro da mente. (CRESWELL, 2007).

Do ponto de vista epistemológico o conhecimento constrói-se através da ação e é situado num contexto. A ação é a chave do pragmatismo, dado que o pensamento está intrinsecamente ligado à ação. Por isso, a realidade e o mundo alteram-se através da ação (KAUSHIK; WALSH, 2019). Por sua vez, as ações relacionam-se com as consequências e, por conseguinte, são passíveis de mudança (MORGAN, 2014). Dependendo das visões que se tem sobre o mundo, de crenças que são socialmente partilhadas, as experiências que cada um tem são diferentes, havendo, no entanto, vários aspetos dessas experiências que são partilhados (MORGAN, 2014). 
No paradigma pragmatista, o problema de investigação é o foco central que determina os métodos e as abordagens. Carateriza-se por uma epistemologia relacional, significando que as elações na investigação são definidas pelo que é apropriado num dado estudo, segundo o investigador (KIVUNJA; KUYINI, 2017). Está, por conseguinte, aberto a uma pluralidade de métodos, quer qualitativos, quer quantitativos. Enquanto paradigma de investigação privilegia a resolução de problemas práticos e valoriza as soluções com impacte na prática (KALOLO, 2015). Estas soluções pretendem resultar em benefícios e nesse sentido não são independentes de valores. Do ponto de vista do pragmatismo, o conhecimento científico é útil quando ajuda as pessoas a lidar melhor com o mundo ou a criar melhores organizações.

\section{Design-Based Research}

\subsection{Raízes epistemológicas}

Design-Based Research é uma metodologia que se tem desenvolvido ao longo das três últimas décadas. Teve origem nos trabalhos de Brown (1992) que, abandonando os métodos positivistas de investigação em Educação, enveredou por um estudo enraizado no contexto, de natureza ideográfico, com uma componente essencial de intervenção prática, baseada num design aplicado e avaliado ao longo do processo investigativo. Brown apelidou-o de design experimentation e refere-se ao estudo realizado dizendo "As a design scientist in my field, I attempt to engineer innovative educational environments and simultaneously conduct experimental studies of those innovations" (BROWN, 1992, p. 141).

O estudo realizado foi influenciado pela filosofia educacional de Dewey, no que se referiu à criação de um cenário de aprendizagem com foco na ação dos alunos, e por Jerome Brunner e Leo Vigotsky, entre outros, relativamente às teorias que nortearam a intervenção, numa perspetiva construtivista.

Desde então, vários investigadores procuraram desenvolver estudos em Educação, com um cariz intervencionista semelhante, com várias denominações - Design studies; Design experiments; Developmental research; Formative research - atestando a crescente adesão a uma investigação com foco na prática e que se revia na procura de alterações visíveis na aprendizagem (AKKER et al., 2006). Wang e Hannafin (2005) entendem que a maioria dos estudos que estão na base desta terminologia se integram numa abordagem 
de Design-Based Research, pois, embora com algumas diferenças nos métodos usados, partilham os mesmos objetivos.

Barab e Squire (2004) referem que a DBR engloba um conjunto de abordagens com o objetivo de produzir novas teorias, artefactos e práticas com impacto na aprendizagem em contextos naturais (p.2). Easterday, Lewis e Gerber (2018), por sua vez, consideram a DBR como uma meta-metodologia segundo a qual investigadores procuram encontrar soluções baseadas na prática para diversos problemas, através de intervenções e modelos de design implementados e sistematicamente avaliados.

Refletindo a crescente importância da tecnologia na sociedade atual, Zheng (2015), refere que a maioria das investigações DBR entre 2004 e 2013 esteve associada a inovações com base em intervenções tecnológicas.

\subsection{Traços distintivos}

Em 2003, vários investigadores associaram-se no Design-Based Research Colective e publicaram um artigo onde propuseram o termo Design-Based Research (DBR) para caracterizar uma emergente metodologia de investigação sobre a aprendizagem em contexto, com o recurso ao design sistemático e estudo de estratégias e ferramentas instrucionais (THE DESIGN-BASED RESEARCH COLECTIVE, 2003). "We use the phrase design-based research methods deliberately (...) to avoid invoking mistaken identification with experimental design, with studies of designers, or with trial teaching methods" (p.1).

Os mesmos autores caracterizaram este tipo de investigação com base em cinco elementos (THE DESIGN-BASED RESEARCH COLECTIVE, 2003):

1. A ligação entre o desenho de ambientes de aprendizagem e o desenvolvimento de "proto teorias" da aprendizagem;

2. A investigação desenvolve-se através de ciclos contínuos de design, implementação, análise e redesign;

3. A investigação sobre o design deverá conduzir a teorias que possam apresentar implicações relevantes para os profissionais no terreno e para outros designers;

4. Os resultados da investigação devem incidir na forma como os designs investigados funcionam em contextos reais; ou seja, não basta documentar 
sucessos ou insucessos, é importante focar os elementos que aprofundam o nosso conhecimento sobre a problemática da aprendizagem em análise;

5. O desenvolvimento destes aspetos deve basear-se em métodos que possam relacionar os processos com os resultados.

Apesar do aumento do número de investigações que se reclamam da metodologia de Design-Based Research (ANDERSON; SHATTUCK, 2012, ZHENG, 2015) não há um consenso sobre as fases ou etapas do processo de investigação.

Todavia, há um conjunto de ideias força que norteiam a DBR enquanto abordagem (AKKER et al., 2006, BARAB, 2004, BARAB; SQUIRE, 2004, COLLINS; JOSEPH; BIELACZYC 2004, WANG; HANNAFIN, 2005, ANDERSON; SHATTUCK, 2012, PETERSON; HERRINGTON, 2005, SHATTUCK; ANDERSON, 2013, BANNANRITLAND, 2003):

- Intervencionista: aplicação de intervenções em situações reais.

- Iterativa: desenvolve-se segundo ciclos sucessivos de design, implementação, avaliação e revisão.

- Orientada pelo processo: com foco na análise e melhoramento da intervenção.

- Pragmática: aspira a alterar a prática; o mérito do design repousa na sua praticabilidade em contextos reais.

- Enraizada na teoria: o design é baseado em premissas teóricas e os resultados contribuem para o avanço da teoria.

- Interativa: investigadores e profissionais da área colaboram na investigação.

Um dos princípios fundamentais da DBR é a conceção de um design que é sucessivamente implementado, em contexto naturalista, de modo a produzir soluções e princípios que possam ser úteis a outros investigadores e profissionais (AMIEL; REEVES, 2008).

Partindo de um problema real, a DBR é sensível ao contexto e assume as caraterísticas de uma investigação colaborativa, onde investigadores e profissionais são coautores do design e do desenvolvimento e análise das sucessivas aplicações de uma intervenção, sujeitas a um refinamento progressivo (Figura 1). Uma intervenção pode focar um artefacto, uma atividade, uma intervenção tecnológica, um sistema ou um processo desenhado com vista à solução do problema, procurando melhorar o conhecimento sobre 
essa intervenção e definindo as condições para a sua melhoria (ANDERSON; SHATTUK, 2012).

Figura 1: Caráter iterativo da DBR

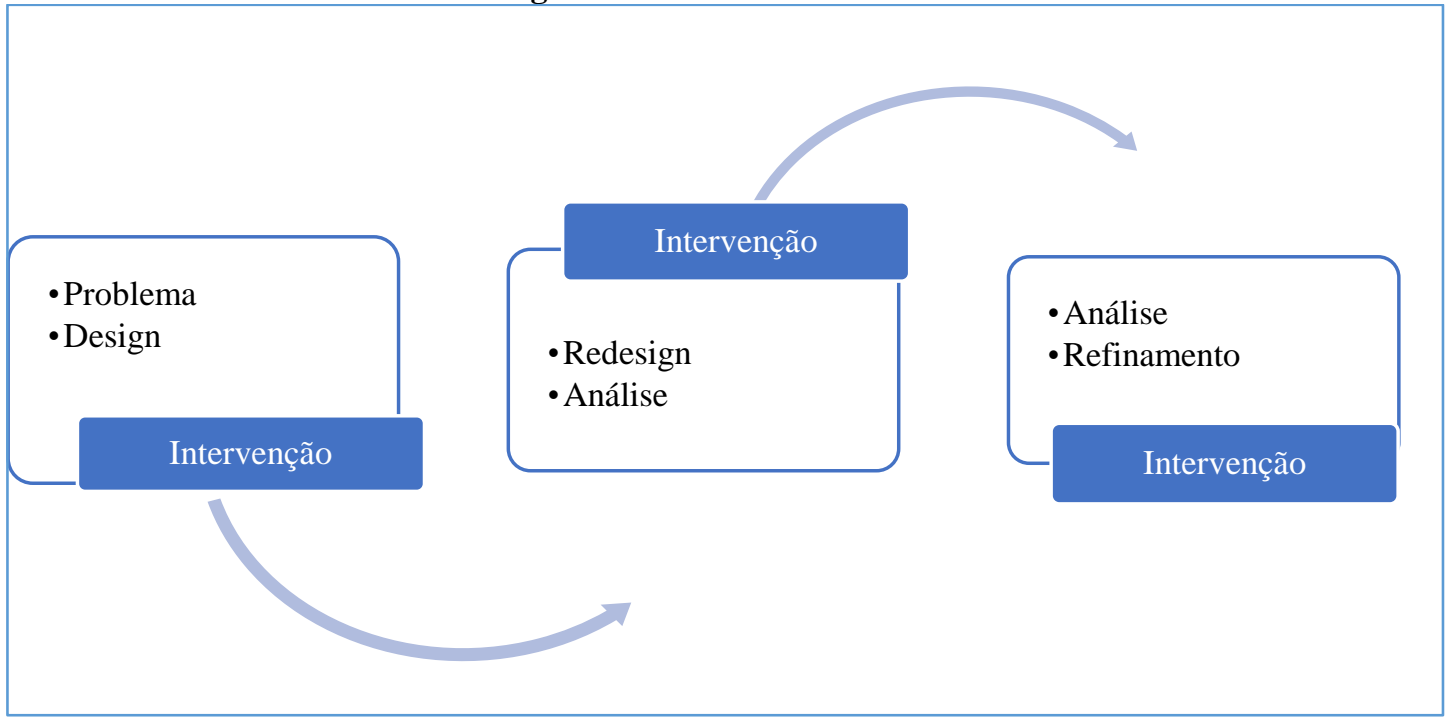

Fonte: As autoras

Os resultados da investigação traduzirão um conjunto de princípios ou guias de ação derivados empiricamente e que, descritos minuciosamente, poderão ser aplicados por outros interessados ao mesmo problema. Embora o objetivo final seja o desenvolvimento da teoria, esse propósito pode exigir múltiplas investigações, testando iterativamente o design com vários grupos (AMIEL; REEVES, 2008). Não se pretende, contudo, o desenvolvimento de grandes teorias, aplicáveis independentemente do contexto. Podem ser princípios de design que enunciam as condições que permitem atingir um objetivo (ANDERSON; SHATTUK, 2012) e modelos clarificando as suas finalidades, os seus elementos e dimensões e os contextos a que se aplicam (EASTERDAY; LEWIS; GERBER 2016). Traduzem, de certo modo, teorias locais, prototeorias no dizer dos investigadores do The Design-Based Research Collective (2003), operacionalizando o foco, a aplicabilidade e a estrutura que carateriza uma nova prática. $\mathrm{O}$ valor das teorias emergentes da investigação reside no seu impacte na prática (BARAB; SQUIRE, 2004).

De acordo com o The Design-Dased Research Collective, esta abordagem metodológica é promissora i) no que respeita à criação de ambientes de aprendizagem inovadores; ii) no desenvolvimento de teorias de instrução e de aprendizagem contextuais; iii) no aprofundamento e consolidação do conhecimento sobre o design em Educação; iv) no incremento da capacidade dos investigadores e profissionais para 
desenvolverem a inovação na educação (DESIGN-BASED RESEARCH COLECTIVE, 2003).

Por sua vez, Cobb, Confrey, diSessa, Lehrer e Schauble (2003) entendem que a DBR é particularmente adequada no desenho de ecologias de aprendizagem, enquanto ambientes (ou artefactos) que incluem problemas e tarefas que os estudantes deverão resolver, normas de discurso e de participação acordadas, recursos e materiais fornecidos e os meios práticos definidos para estabelecer de que modo estes elementos são relacionados pelo professor e estudante. A metáfora da ecologia pretende sublinhar o caráter sistémico e integrador do design, rompendo com uma conceção de listas de atividades e de estudo de fatores isolados.

\subsection{Desafios emergentes}

Um dos desafios da DBR provêm do facto de ser ancorada em contextos naturalistas. A investigação confronta-se com a complexidade do mundo real e a inércia, ou mesmo resistência, à mudança (COLLINS; JOSEPH; BIELACZYC, 2004). Envolve múltiplas variáveis, do ambiente, dos produtos, dos sistemas e dos participantes que povoam as situações, por vezes caóticas e confusas onde ocorre a aprendizagem (BARAB; SQUIRE, 2004). A análise da implementação do design não segue um padrão uniforme, aplicável a todos os estudos deste tipo. Cada estudo, de acordo com a teoria subjacente, os objectivos e o contexto, seleciona os métodos a usar e os instrumentos para recolha de dados, sendo que a própria evolução do design exige novas decisões e novas formas de olhar. Bell (2004), a este propósito, refere que dentro do âmbito das investigações que se posicionam como DBR, se pode considerar a existência de famílias que se inserem em diferentes campos teóricos e que usam métodos de análise diferentes. Por isso, a DBR é pluralista quanto às teorias e quanto métodos, sendo frequente a combinação de métodos quantitativos e qualitativos.

Uma das questões que merece reflexão diz respeito à possibilidade de generalização dos resultados, incluindo os avanços no que se refere às teorias em que se baseiam os estudos. Sendo uma abordagem implementada num contexto, procurando saber o que funciona, comofunciona e porque funciona uma dada inovação num contexto, tendo em conta os factores que o caraterizam (PETERSON; HERRINGTON, 2005), os resultados relativos a uma intervenção não são generalizáveis a outros contextos. Todavia, espera-se que eles sejam testados e aplicados em contextos semelhantes. Ao 
invés de considerar a generalização como uma meta da investigação, procura-se a transferibilidade dos resultados. A implementação iterativa do design respetivo noutros contextos irá proporcionar o refinamento das conclusões, quer em termos concetuais, quer de usabilidade contextual, potenciando a inovação local e o aumento do conhecimento no campo.

The challenge for design-based research is in flexibly developing research trajectories that meet our dual goals of refining locally valuable innovations and developing more globally usable knowledge for the field (THE DESIGNBASED RESEARCH COLLECTIVE, 2003, p. 7).

Sendo a DBR sensível ao contexto, Sandoval (2014) alerta para que uma aplicação do design em grande escala, sem ter em conta as caraterísticas do contexto não é uma forma adequada de procurar aumentar a transferibilidade da inovação desenhada. Sugere que se procurem trajetórias de estudo que envolvam contextos de igual escala, escolhendo-os em função de pontos de partida locais semelhantes. Deste modo, os resultados da implementação do design nesses novos contextos pode ajudar a clarificar os traços que concorrem melhor (ou não concorrem) para atingir os objetivos pretendidos.

Há um consenso entre os investigadores sobre vários aspetos que tornam difícil a aplicação desta metodologia e podem dar origem a limitações. Primeiro, não é possível (nem seria eticamente desejável), manipular contextos culturais e sociais (HOADLEY, 2004), pelo que as situações em estudo se apresentam complexas, com múltiplos fatores internos e/ou externos que tornam difícil as tarefas de recolha e análise de dados, sobretudo com equipas de tamanho reduzido.

Em segundo lugar, uma abordagem DBR origina uma grande quantidade de dados, de natureza diversa. Por esse motivo a triangulação na análise dos dados e das fontes é crucial (THE DESIGN-BASED RESEARCH COLLECTIVE, 2003). Este aspeto pode ser determinante para a credibilidade da investigação.

Em terceiro, sendo os resultados relativos ao contexto em estudo, não é possível replicar da mesma forma um design estudado, pois o novo contexto introduz novos aspetos a ter em conta. A replicação em grande escala a partir de uma amostra reduzida torna-se impossível (BARAB; SQUIRE, 2004; HOADLEY, 2011; O’DONNELL, 2004).

Decorrente destes aspetos, torna-se relevante que o design implementado seja minuciosamente relatado, envolvendo a descrição do contexto, das teorias subjacentes e emergentes do estudo, dos aspetos principais da intervenção, das diversas iterações e do impacte dos diversos aspetos na participação e aprendizagem (BARAB; SQUIRE, 2004) e, ainda dos aspetos negativos ou problemáticos (THE DESIGN-BASED RESEARCH 
COLLECTIVE, 2003). Sandoval (2014) salienta ainda a importância da explicitação das conjeturas feitas pelos investigadores, aquando da conceção e da implementação do design.

Hoadley (2004), face a críticas sobre a validade da investigação DBR, refere que eventuais critérios para avaliar uma investigação em DBR estão ainda em construção. E do seu ponto de vista, há que procurar novas formas de validade. Acrescenta que é necessário assumir a validade da intervenção/processo, entendida como o alinhamento preciso da intervenção com as teorias que a sustentam. Para além desta, haverá que ter em conta a validade sistémica, isto é, a explicitação das teorias que foram testadas no decurso da investigação, no seu todo, e a forma como foram feitas as inferências que provam os resultados. E refere, ainda, a necessidade de definir a validade consequencial, isto é, o modo como as teorias em estudo (ou emergentes) podem ser aplicadas subsequentemente.

Barab e Squire (2004), por sua vez, alertam que a credibilidade de uma investigação DBR reside na importância da validade consequencial, próxima de uma noção de utilidade (usefulness). Esta deriva do compromisso dos investigadores de procurar investigar para alterar a prática, o que, por sua vez, os obriga a serem muito claros no tipo de conclusões que extraem a partir dos resultados e nas limitações dos mesmos.

\section{Investigação-Ação}

\subsection{Raízes epistemológicas}

A investigação-ação (IA) há muito que faz parte da investigação em educação, tal como em outras áreas do campo das ciências sociais. A expressão investigação-ação foi criada em 1940 por Kurt Lewin (1890-1947) para designar formas de investigação que pretendiam resolver problemas sociais. Consistia em planificar, analisar e identificar factos e avaliação precisos e implicava a participação do grupo através de processos democráticos. O termo "ação" foi acrescentado ao termo "investigação" como forma de dar resposta aos desafios verificados na melhoria das relações de grupo.

A IA significa ação por parte da organização em consideração e das pessoas envolvidas na organização (MCNIFF, 1991). Neste sentido, implica envolvimento e melhoramento educacional. Identificado o problema e a área onde se vai desenvolver a 
ação reconhece-se que a resolução da situação problemática, baseada em negociação e participação, terá repercussões em aspetos alargados da organização escolar.

Nas suas diversas formas, a IA é um conjunto de compromissos, uma metodologia e não um conjunto de técnicas (um método) para a investigação. Incorpora várias epistemologias, ou seja, formas variadas de gerar conhecimento. Embora as estratégias de recolha de dados e as abordagens que orientam a análise de dados nas várias formas de IA sejam variadas, todas partilham uma epistemologia que vê o conhecimento como essencialmente conectado à prática - a epistemologia da prática (NOFFKE, 1997).

A literatura sobre a IA dá conta de diferenças ao nível dos processos e dos propósitos da investigação (CARR; KEMMIS, 1986; MCKERNAN, 1996; KEMMIS; MCTAGGART, 2005; MCNIFF; WHITEHEAD, 2010; ANDERSON; HERR, 2010; NOFKE; SOMERKH, 2010). Existem novas estratégias de recolha de dados e respetiva análise que se prendem com as abordagens teóricas subjacentes à investigação a par de outras formas analíticas mais "tradicionais". Há, por exemplo, estudos que se focam no trabalho desenvolvido em sala de aula, no desenvolvimento profissional ou organizacional (ANDERSON; HERR, 2010; OLIVEIRA; HENRIQUES, 2017) enquanto outros têm subjacente uma visão social alargada e procuram ligações a movimentos sociais.

\subsection{Traços distintivos}

Em geral, aceita-se que a IA é inquiry (no sentido de Dewey) movido por ou com insiders de uma organização ou comunidade mas nunca para ou sobre eles. O inquiry sistemático é público (MCNIFF, 1991; STENHOUSE, 1980), observa e descreve o que está a acontecer e explica, ou seja, diz porquê e como. É orientada para a ação ou ciclos de ações que os membros de uma organização ou comunidade desenvolvem em torno de uma situação problemática. A mudança ocorre no contexto e/ou nos próprios investigadores. A IA é enraizada em valores, tal como todas as formas de inquiry, ou seja, tem lugar num cenário que reflete uma sociedade caracterizada por conflitos entre valores. É um processo reflexivo, distinto de uma reflexão isolada, espontânea, uma vez que é deliberado e sistemático e requer evidência para suportar as asserções. A noção de reflexividade é crucial (BODGAN; BIKLEN, 1994; WELLINGTON, 2000) porque os investigadores devem indagar sobre formas anteriores de melhoria ou soluções encontradas considerando quem beneficiou das ações que foram levadas a cabo. 
Em todos os contextos as questões em investigação-ação emergem das práticas no domínio da investigação académica e do mundo do trabalho, nomeadamente em escolas. As intenções e as metodologias tornam-se evidentes quando os grupos orientam a IA para o desenvolvimento pessoal e profissional, a produção de conhecimento e na ótica de movimentos sociais (NOFKE, 2002).

O método da IA envolve uma espiral de planeamento, ação, observação, reflexão e re-planeamento, o que exige conhecimento do que foi feito anteriormente pelos profissionais. Habitualmente são usadas espirais para representar o processo sublinhandose que a IA oferece a possibilidade de tratar mais do que um problema ao mesmo tempo, na medida em que um problema pode ter a ver com outros subjacentes. Na figura 2 apresentam-se três ciclos relevando que o primeiro passo do $2^{\circ}$ ciclo emerge com a redefinição do problema e o consequente replaneamento da ação.

Figura 2: Modelo de investigação-ação - representação de três ciclos

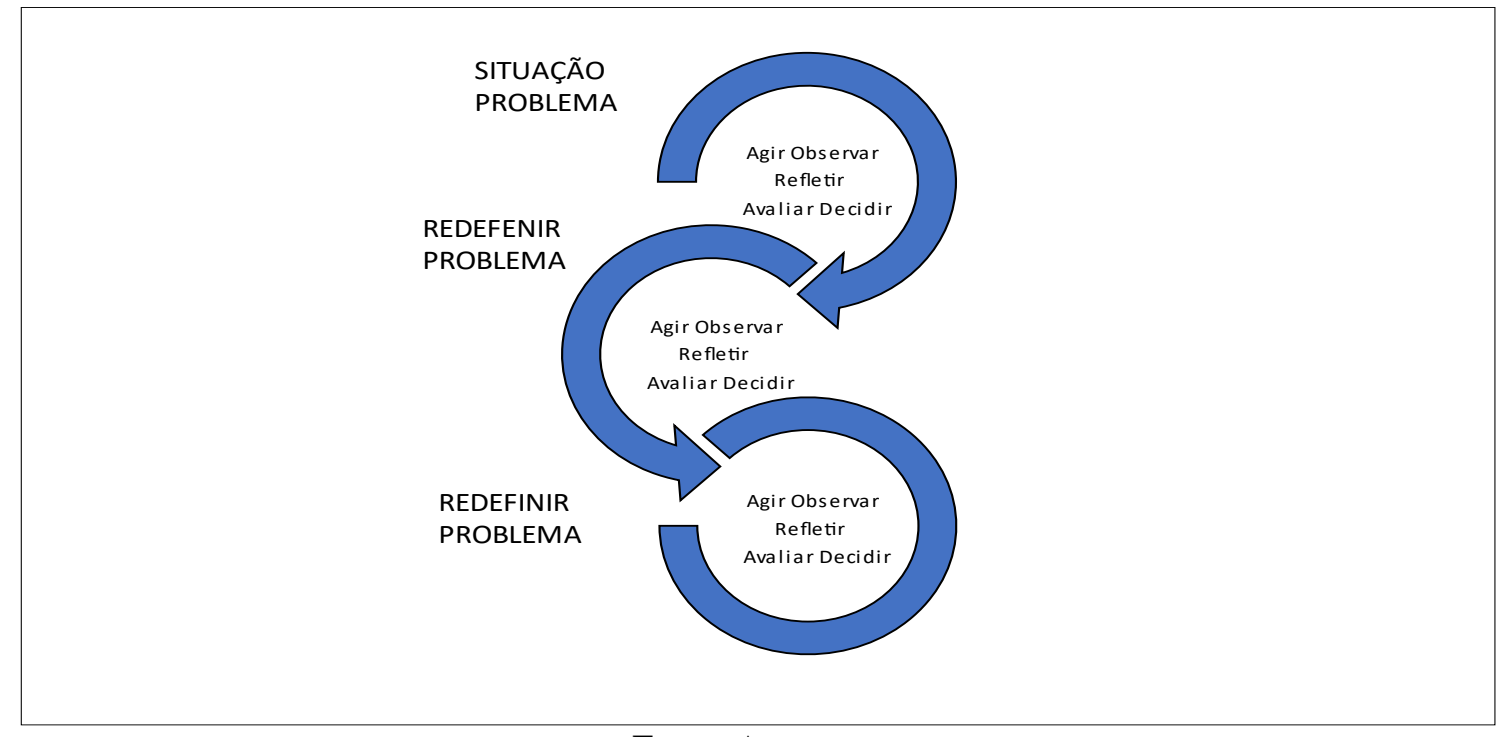

Fonte: As autoras

Embora exista uma diversidade de representações do processo de IA, os ciclos de atividades seguem, em geral, uma série de passos que consistem em:

1. Desenvolver um plano de ação para melhorar o que está a acontecer;

2. Agir para implementar o plano;

3. Observar os efeitos da ação no contexto em que ocorre;

4. Reflectir nesses efeitos como base para planeamento adicional, ação subsequente e novos ciclos. Deste modo, cada ciclo aumenta o conhecimento do problema original e, espera-se, que conduza à sua solução. 


\subsection{Desafios emergentes}

Há um leque de questões que a IA suscita, desde logo, as que se prendem com a validade (CUNHA; FIGUEIREDO, 2001; MCNIFF, 1991; NEWTON; BURGESS, 2008). A este propósito, McNiff (1993) e Kemmis e McTaggart (2005) propõem o seguinte questionamento: Perante as diversas interpretações quais as que têm sentido para os investigadores e para os outros, ou seja, até que ponto são compreensíveis? Dessas interpretações quais as consideradas verdadeiras, precisas, tendo em conta o que é conhecido? E ainda, quais as que são sustentadas e descritas no sentido da autenticidade das mesmas. E dadas as circunstâncias em que foram produzidas, quais são as moralmente corretas e apropriadas?

Anderson e Herr (1999) definem um conjunto de critérios de validade que consideram experimentais e em fluxo, a saber: validade dos resultados que se refere à extensão em que os resultados da pesquisa correspondem aos propósitos pretendidos da pesquisa; validade do processo relacionada com a eficácia da abordagem da investigação ao problema a investigar; validade democrática que se prende com a investigação ser realizada em colaboração com todas as partes interessadas no problema sob investigação; validade catalítica refere-se à capacidade do processo de investigação em transformar os participantes, aprofundar a compreensão dos participantes e motivá-los para a promoção de ações sociais; e a validade dialógica refere-se a se os profissionais participem do diálogo crítico e reflexivo uns com os outros.

No Quadro 1 é apresentada a proposta de Herr e Anderson (2005) sobre a ligação entre as diversas metas da IA e os critérios de validade por eles identificados.

\begin{tabular}{|l|l|}
\hline \multicolumn{2}{|c|}{ Quadro 1: Metas da investigação-ação e critérios de validade } \\
\hline Metas da investigação-ação & \multicolumn{1}{c|}{ Critérios de validade } \\
Corar novo conhecimento & Validade dialógica e de processo \\
Educar investigador e participantes & Validade de resultado \\
Alcançar resultados relevantes para o contexto local & Validade catalítica \\
Metodologia de investigação sólida e apropriada & Validade democrática \\
\hline
\end{tabular}

Fonte: Adaptado de Herr e Anderson (2005, p. 55)

A multiplicidade de estudos que se reclama de IA e a forma como são sistematizados e elaborados modelos constitui outra questão relevante que tem merecido reflexão por parte de diversos autores (MCKERNAN, 1999; NEWTON; BURGESS, 
2008; NOFFKE, 1997, 2002). McKernan (1999) considera três tipos ou modelos teóricos do processo de IA. A IA científica que se aproxima do método científico de resolução de problemas, onde inclui os estudos de Lewin (1947). A IA prático-deliberativa (STENHOUSE, 1984; ELLIOT, 1991) cujo propósito é compreender a prática e resolver problemas, sendo que a deliberação responde a uma situação problemática do ponto de vista moral. A IA crítico-emancipatória (CARR; KEMMIS, 1986; KEMMIS; TAGGART, 1994) rejeita a perspetiva positivista no papel instrumental do conhecimento na solução de problemas e advoga que os profissionais não só ressignificam a ação mas também a organizam. A este modelo subjaz o que Habermas $(1987,2002)$ designa como interesses constitutivos do conhecimento, a saber: prático e emancipador, trazendo para a ciência a hermenêutica, a crítica assente em espirais reflexivas da ação humana.

Susan Noffke (1997, 2002) com base na análise das distintas abordagens, propósitos e práticas de IA propõe três dimensões - profissional, pessoal e política - sem hierarquização e refletindo diferentes ênfases, uma vez que todas as formas de IA incorporam uma dimensão política. Na dimensão profissional Noffke (2002) inclui os movimentos que no séc XX, em campos emergentes, como a educação, em que a IA surge como uma atividade de "geração de conhecimento". Inclui os trabalhos de Lawrence Stenhouse (1984) e John Elliott (1991), no Reino Unido, que foram desenvolvidos no sentido de reformular a natureza do ensino como uma forma de investigação. Construíram um corpo de conhecimento sobre a prática educativa e uma conceção de ensino centrada na reflexão sobre dados da própria prática. Segue, também, esta perspetiva o movimento 'teacher research', associado ao desenvolvimento profissional dos professores, onde se destacam Marilyn Cochran-Smith e Susan Lytle.

Na dimensão pessoal NoffKe (2002) inclui estudos em que a IA tem impacto no crescimento e desenvolvimento pessoal dos que se envolvem nela; outros que enfatizam a natureza individual versus a colaboração do trabalho; e ainda os abordam o envolvimento de professores universitários individuais no processo de IA. O objetivo nestes casos é que os profissionais participem no processo de geração de conhecimento. Enquadra-se nesta dimensão o "self-study" baseado nas histórias de vida e nas narrativas pessoais, que se desenvolveu na formação de professores, desde o início dos anos 90. Destacam-se os trabalhos de Jack Whitehead e Jean NcNiff, nos anos 90 do séc XX, em que o primeiro autor desenvolve as 'living educational theories' geradas da prática.

Na dimensão política há um propósito central na IA - a procura de soluções para problemas sociais. O uso da IA para promover mudanças sociais, ao nível das 
desigualdades sociais e no âmbito dos movimentos culturais e feministas, entre outros. Em educação, enquadram-se nesta dimensão o trabalho de Paulo Freire, de Henry Giroux com a pedagogia crítica e de outros investigadores que se apoiam na teoria crítica têm tido influência na IA em educação. Com o trabalho de Carr e Kemmis (1986) que convocam a teoria crítica de Jürgen Habermas sobre o potencial em transformar a prática profissional há novos desenvolvimentos na IA, considerando a dimensão política.

As questões da validade têm acompanhado os múltiplos estudos em IA, conduzindo a reflexões que procuram uma sistematização, tal como a apresentada por Newton e Burgess (2008) que a carateriza como emancipatória, prática e geradora de conhecimento. Esta caraterização sustenta-se na ênfase atribuída às diferentes validades definidas por Anderson \& Herr's (1999), as quais dependem dos propósitos e epistemologias da investigação. Para estes autores o tipo de IA que se pretende desenvolver demarca a configuração de validade para avaliar a reivindicação de conhecimento num dado projeto.

\section{Convergências}

A comparação entre as duas abordagens que foram objeto anterior de análise faz emergir uma grande aproximação entre elas (Quadro 2).

Quadro 2: Comparação entre DBR e IA

\begin{tabular}{|l|l|l|}
\hline & \multicolumn{1}{|c|}{ IA } & \multicolumn{1}{c|}{ DBR } \\
\hline Propósitos & $\begin{array}{l}\text { Melhorar as práticas profissionais } \\
\text { Empoderar os profissionais } \\
\text { Gerar conhecimento }\end{array}$ & $\begin{array}{l}\text { Alterar a prática } \\
\text { Refinar teoria } \\
\text { Produzir teorias locais }\end{array}$ \\
\hline Foco & Ação mediada & $\begin{array}{l}\text { Criação e análise de um design na } \\
\text { prática }\end{array}$ \\
\hline Conceitos chave & $\begin{array}{l}\text { Inquiry } \\
\text { Melhoria } \\
\text { Participação } \\
\text { Colaboração } \\
\text { Reflexividade } \\
\text { Ciclos em espiral }\end{array}$ & $\begin{array}{l}\text { Iteração (ciclos iterativos) } \\
\text { Interação (colaboração) }\end{array}$ \\
Design \\
Contexto \\
Análise \\
Teorias locais \\
Inovação
\end{tabular}




\begin{tabular}{|l|l|l|}
\hline Técnicas & Escalas de atitudes & Listas de verificação \\
Questionários \\
Entrevistas \\
Narrativas \\
Testes
\end{tabular}

Fonte: As autoras

Ambas são orientadas para a ação. Se no caso da IA, as questões de investigação emergem da própria prática, no caso da DBR é a procura de encontrar novas soluções para a prática que a orientam o investigador. Ambos os modelos de investigação partilham a necessidade de a investigação se realizar em situações e contextos reais. A IA aspira à produção do conhecimento sobre a própria prática. Por sua vez, a DBR, partindo de premissas teóricas, aspira a que os resultados da investigação possam aprofundar o conhecimento sobre o modelo de intervenção desenhado e possam gerar novas teorias sobre os problemas em estudo.

A IA enfatiza a reflexão sobre os dados da prática e procura observar os efeitos da ação no contexto em que ocorrem. De modo similar, na DBR é a análise dos processos ocorridos na situação real que orienta o redesenho da intervenção de modo a alcançar resultados úteis.

Ambas as abordagens partilham um cunho colaborativo, onde investigadores e profissionais partilham os objetivos da investigação e combinam os seus esforços no decurso da mesma.

Finalmente, quer a IA quer a DBR consideram a produção do conhecimento é indissociável da prática, quer numa epistemologia da prática (IA), quer no valor e utilidade da teoria para a prática (DBR).

\section{Síntese final}

A aproximação ao pragmatismo tem a ver com o facto da IA e da DBR terem um design de investigação consolidado que usa uma pluralidade de métodos e que se desenvolve por uma busca de subquestões ou refinamento progressivo, partindo dos contextos em que o investigador está a estudar. São investigações enraizadas em valores 
orientados para a utilidade das consequências, aspirando a melhorar as práticas (IA) ou a construir novas práticas (DBR).

Do ponto de vista ontológico não consideram a existência de uma realidade única, imutável, e assumem a perspetiva de que o conhecimento é provisório, sensível à alteração do contexto. Esta assunção justifica a existência dos ciclos contínuos na IA e da implementação iterativa do design na DBR.

Do ponto de vista epistemológico, ambas as abordagens assentam na ideia de que o conhecimento é construído na interseção das teorias com os contextos. Por isso, uma vez que as situações e os contextos são complexos, sujeitos a múltiplos olhares e variações, abordam a investigação de um ponto de vista colaborativo, englobando investigadores e profissionais no terreno. Dito de outro modo, a IA e a DBR rompem com o investigador isolado da prática (outsider), privilegiando a procura de soluções sustentáveis com consequências imediatas para ação.

Do ponto de vista metodológico, as questões de investigação são enfatizadas, através de subquestões ou refinamento nas mesmas, e tanto a IA como a DBR partilham um caminho próprio, que assenta em ciclos/iterações contínuos ou evoluindo para novos ciclos (IA) ou para (re)desenhos em contextos similares (DBR). Neste processo, não há um método ou técnica fixa ou única, pelo que os estudos nestas duas abordagens são abertos a uma pluralidade de opções, selecionadas em função dos objetivos de investigação.

Estas semelhanças, muito focadas na valorização da prática como meio de produzir conhecimento, e na utilidade das consequências dos resultados da investigação para ação, colocam ambas as metodologias num paradigma de investigação em Educação de natureza pragmática.

Refletindo sobre as caraterísticas da DBR e da AR, entendemos que o que as aproxima se centra na perspetiva da investigação para e na ação, na utilidade dos resultados para a educação, quer para o desenvolvimento profissional e organizacional a exemplo da IA, quer para a construção de cenários inovadores e novos artefactos no caso da DBR, na utilização de abordagens múltiplas e métodos diversificados e na perspetiva colaborativa para melhor encontrar soluções credíveis e funcionais para os problemas. Estes traços, do nosso ponto de vista, levam-nos a enquadrar ambas as abordagens num Paradigma Pragmático de Investigação em Educação. Se no caso da DBR, esta assunção é inclusive partilhada com alguns autores (BARAB; SQUIRE, 2004, WANG; HANNAFIN, 2005), no caso da IA é uma assunção que contrasta com outras visões 
(COHEN; MANION; MORRISSON, 2004), que colocam alguns estudos nesta abordagem como fazendo parte do Paradigma Crítico. Contudo, a IA na investigação em educação enfatiza a participação democrática não estando dependente de uma teoria. No processo de inquiry que é central em IA e na análise dos dados destaca-se o compromisso com a melhoria educacional e os racionais teóricos que emergem da ação, a sustentam e ampliam. Nesta perspetiva, não se pretende rejeitar a IA que usa a teoria crítica, antes se argumenta que ao integrá-la no paradigma crítico se está a condicionar parte da IA realizada em educação. É nossa convicção que, tendo em conta o quadro acima traçado, ambas as metodologias se situam no paradigma pragmático.

\section{Referências}

AKKER, J. V. D et al. Introduction to educational design research. In: AKKER, J. V. D. (ed.). Educational design research. New York: Routledge, 2006. p. 3-7.

COLLINS, A.; JOSEPH, D.; BIELACZYC, K. Design Research: Theoretical and Methodological Issues. Journal of the Learning Sciences, Philadelphia, v.13, n. 1, p 15-42, novembro. 2004.

AMIEL, T.; REEVES, T. C. Design-Based Research and Educational Technology: Rethinking Technology and the Research Agenda. Educational Technology \& Society, Taiwan, v.11, n.4, October. 2008.

ANDERSON, G. L.; HERR, K. The new paradigm wars: Is there room for rigorous practitioner knowledge in schools and universities? Educational Researcher, Washington, v. 28, n. 5, p. 12-21, june. 1999.

ANDERSON, T.; SHATTUCK, J. Design-Based Research: A Decade of Progress in Education Research? Educational Researcher, Washington, v. 41, n. 1, p. 16-25, jan./feb. 2012.

BANNAN-RITLAND, B. The Role of Design in Research: The Integrative Learning Design Framework. Educational Researcher, Washington, v. 32, n. 1, p. 21-24, january. 2003. BARAB, S.; SQUIRE, K. Design-Based Research: Putting a Stake in the Ground. Journal of the Learning Sciences, Philadelphia, v. 13, n. 1, p. 1-14, nov. 2004.

BARAB, S.A. Using Design to Advance Learning Theory or Using learning Theory to Advance Design. Educational Technology, Englewood Cliffs, v. 44, n. 3, p. 16-20, may./june. 2004.

BELL, P. On the Theoretical Breadth of Design-Based Research in Education. Educational Psychologist, Philadelphia, v. 39, n. 4, p. 243-253, june. 2004.

BODGAN R.C.; BIKLEN, S. K. Investigação Qualitativa em Educação - Uma Introdução à Teoria e aos Métodos. Porto: Porto Editora, LDA, 1994.

BROWN, A. L. Design Experiments: Theoretical and Methodological Challenges in Creating Complex Interventions in Classroom Settings. The Journal of the Learning Sciences, Philadelphia, v. 2, n. 2, p.141-178. 1992. 
CARR, W.; KEMMIS, S. Becoming critical: education, knowledge and action research. London: Routledge Falmer, 1986.

COBB, P. et al. Design Experiments in Educational Research. Educational Researcher, Washtington, v. 32, n. 1, p. 9-13, jan. 2003.

COHEN, L.; MANION, L.; MORRISSON, K. Research Methods in Education. 5th ed. London: RoutledgeFalmer, 2004.

CRESWELL, J. W. Qualitative inquiry and research design: choosing among five traditions. 1st ed. London: SAGE Publications, 1998.

CRESWELL, J. W. Research Design. Qualitative, Quantitative and Mixed Methods Approaches. 2nd ed. London: SAGE Publications, 2003.

CRESWELL, J. W. Qualitative Inquiry Research Design: Choosing Among Five Approaches. 2nd ed. USA: Sage Publications, 2007.

CUNHA, P. R.; FIGUEIREDO, A. D. Action-research and critical rationalism: A virtuous marriage. In: EUROPEAN CONFERENCE ON INFORMATION SYSTEMS, INFORMATION SYSTEMS AND THE FUTURE OF THE DIGITAL ECONOMY, 10., 2002, Gdansk, Poland. Proceedings of the 10th European Conference on Information Systems, Information Systems and the Future of the Digital Economy, ECIS 2002. Gdansk, 2002. p. 19-27. Disponível em: https://pdfs.semanticscholar.org/40ec/6da54cc1d0d2038e3a44268dd5b26e53d99c.pdf. Acesso em: 13 ago. 2021.

DENZIN, N. K.; LINCOLN, Y. S. Handbook of qualitative research. Thousand Oaks, CA: SAGE, 1994.

DENZIN, N. K.; LINCOLN, Y. S. Handbook of qualitative research. 2nd ed. Thousand Oaks, CA: SAGE, 2000.

EASTERDAY, M.W.; LEWIS, D. G. R.; GERBER, E. M. The logic of the theoretical and practical products of design research. Australasian Journal of Educational Technology, Tugun, v. 32, n. 4, p.125-144. 2016.

ELLIOTT, J. Action research for educational change. UK: Open University Press, 1991.

GASPAR, M. I. et al. Modelos para ensinar - escolhas do professor. Lisboa: Chiado Editora, 2015.

GUBA, E.; LINCOLN, Y. Paradigmatic controversies, contradictions, and emerging confluences. In: DENZIN, N.; LINCOLN, Y. (ed.). Handbook of qualitative research. 3. ed. Thousand Oaks, CA: SAGE, 2005. p. 191-215.HABERMAS, J. Théorie de l'agir communicationnel (Vol. II). Poitiers: Fayard, 1987. [Original publicado em alemão em 1981].

HABERMAS, J. Racionalidade e comunicação. Lisboa: Edições 70, 2002.

HERR, K.; ANDERSON, G.L. The action research dissertation: a guide for students and faculty. Thousand Oaks: Sage Publications, 2005.

ANDERSON, G. L.; HERR, K. Practitioner Action Research and Educational Leadership. In: NOFFKE, S. E.; SOMEKH, B. (ed.). The Sage Handbook of Educational Action Research. London: SAGE, 2010. p. 154-166. 
IOANNIDOU-KOUTSELINI, M.; PATSALIDOU, F. Engaging school teachers and school principals in an action research in-service development as a means of pedagogical selfawareness. Educational Action Research, Nottingham, v. 23, n. 2, p. 124-139, mar. 2015.

KALOLO, J. F. The Drive towards Application of Pragmatic Perspective in Educational Research: Opportunities and Challenges, Journal of Studies in Education, Las Vegas, v. 5, n. 1, p. 150-171. february. 2015.

KIVUNJA, C.; KUYINI, A.B. Understanding and Applying Research Paradigms in Educational Contexts. International Journal of Higher Education, Richmond Hill, v. 6, n. 5, p. 26-41. october. 2017.

KAUSHIK, V.; WALSH, C.A. Pragmatism as a Research Paradigm and Its Implications for SocialWork Research. Social Sciences, Basel, v. 8, p. 255: 1-17, sep. 2019.

KEMMIS, S.; MCTAGGART, R. Participatory action research. In: DENZIN, N. K.; LINCOLN, Y. S. (ed.). Handbook of qualitative research. Thousand Oaks, CA: Sage Publications, 1994. p. 567-605.

KEMMIS, S.; WILKINSON, M. Participatory action research and the study of practice. In: ATWEH, B.; KEMMIS, S.; WEEKS, P. (ed.). Action research in Practice - partnerships for Social Justice in Education. London: Routledge, 1998. p. 21-36.

LINCOLN, Y. S.; GUBA, E. G. Paradigmatic controversies, contradictions, and emerging confluences. In: DENZIN, N. K.; LINCOLN, Y.S. 2nd ed. Handbook of qualitative research, London: Sage, Chapter 6, 2000. p. 163-188.

MCKERNAN, J. Investigación-acción y curriculum. Madrid: Morata, 1996.

MCNIFF, J. Teaching as learning: an action research approach. London: Routledge, 1993.

MCNIFF, J.; LOMAX, P.; Whitehead, J. You and your action research project. London: Routledge, 1996.

MORGAN, D. L. Paradigms Lost and Pragmatism Regained: Methodological Implications of Combining Qualitative and Quantitative Methods. Journal of Mixed Methods Research, Indianapolis, v. 1, n. 1, p. 48-76, jan. 2007.

MORGAN. D. L. Pragmatism as a Paradigm for Social Research. Qualitative Inquiry, v. 20, n. 8, p. 1045-1053, oct. 2014.

NEWTON, P.; BURGESS, D. Exploring types of educational research: implications for research e validity. International Journal of Qualitative Methods, Alberta, v. 7, n. 4, p. 1830, dec. 2008.

NOFFKE, S. E. Action research: towards the next generation. In: DAY, C. et al. (Eds.) Theory and practice in action research: some international perspectives. Oxford: Symposium Books, 2002. p. 13-26.

NOFFKE, S. E. Professional, personal, and political dimensions of action research. Review of Research in Education, Washington, v. 22, n. 1, p. 305-343, jan. 1997.

OLIVEIRA, I.; HENRIQUES, S. (org.). Investigação ação em práticas de liderança educacional. Lisboa: Universidade Aberta. eUAB Coleção Universitária, 2017. 
PEREIRA, A.; OLIVEIRA, I. Design-based research e investigação-ação: dois olhares que se cruzam. News Trends in Qualitative Research, [S. l.], v. 2, p. 336-350, 2020. DOI: 10.36367/ntqr.2.2020.336-350. Disponível em: https://publi.ludomedia.org/index.php/ntqr/article/view/. Acesso em: 03 ago. 2021.

PETERSON, R.; HERRINGTON, J. The State of the Art of Design-Based Research. In: WORLD CONFERENCE ON E-LEARNING IN CORPORATE, GOVERNMENT, HEALTHCARE, AND HIGHER EDUCATION, 24, 2005, Vancouver. Proceedings of ELearn 2005--World Conference on E-Learning in Corporate, Government, Healthcare, and Higher Education. Vancouver, Canada: Association for the Advancement of Computing in Education (AACE), 2005. p. 2302-2307. Disponível em https://researchrepository.murdoch.edu.au/id/eprint/5250/. Acesso em: 13 jul. 2021.

SANDOVAL, W. Conjecture Mapping: An Approach to Systematic Educational Design Research. Journal of the Learning Sciences, Philadelphia, v. 23, n. 1, p. 18-36, apr. 2014. SHATTUCK, J.; ANDERSON, T. Using a Design-Based Research Study to Identify Principles for Training Instructors to Teach Online. The International Review of Research in Open and Distributed Learning, Athabasca, v. 14, n. 5, p.1-22, dec. 2013.

STENHOUSE, L. Investigación y desarrollo del curriculum. Madrid: Ediciones Morata, S.L, 1984.

THE DESIGN- BASED RESEARCH COLLECTIVE. Design-Based Research: An Emerging Paradigm for Educational Inquiry. Educational Researcher, Washington, v. 32, n. 1, p. 5-8, 2003.

WANG, F.; HANNAFIN, M. J. Design-Based Research and Technology-Enhanced Learning Environments. Educational Technology Research and Development, New York, v. 53, n. 4, p. 5-23. 2005.

WELLINGTON, J. Educational Research - Contemporary Issues and Practical Approaches. London: Continuum, 2000.

ZHENG, L. A systematic literature review of design-based research from 2004 to 2013. J. Comput. Educ., Beijing, v. 2, n. 4, p. 399-420, dec. 2015.

Recebido em: 15 de julho de 2021.

Aceito em: 20 de agosto de 2021. 